\title{
BMJ Open Factors associated with prolonged length of stay following cardiac surgery in a major referral hospital in Oman: a retrospective observational study
}

\author{
Ahmed Almashrafi, ${ }^{1}$ Hilal Alsabti, ${ }^{2}$ Mirdavron Mukaddirov, ${ }^{2}$ Baskaran Balan, ${ }^{2}$ \\ Paul Aylin ${ }^{1}$
}

To cite: Almashrafi $A$, Alsabti H, Mukaddirov M, et al. Factors associated with prolonged length of stay following cardiac surgery in a major referral hospital in Oman: a retrospective observational study. BMJ Open 2016;6:e010764. doi:10.1136/bmjopen-2015010764

- Prepublication history for this paper is available online. To view these files please visit the journal online (http://dx.doi.org/10.1136/ bmjopen-2015-010764).

Received 4 December 2015 Revised 5 February 2016 Accepted 19 April 2016

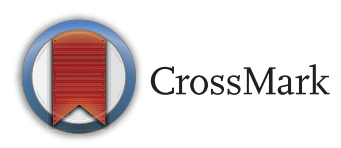

${ }^{1}$ Department of Primary Care and Public Health, School of Public Health, Imperial College London, London, UK ${ }^{2}$ Cardiothoracic Surgery Division, Department of Surgery, Sultan Qaboos University Hospital, Muscat, Oman

Correspondence to Ahmed Almashrafi; a.almashrafi12@imperial.ac.uk

\section{ABSTRACT}

Objectives: Two objectives were set for this study. The first was to identify factors influencing prolonged postoperative length of stay (LOS) following cardiac surgery. The second was to devise a predictive model for prolonged LOS in the cardiac intensive care unit (CICU) based on preoperative factors available at admission and to compare it against two existing cardiac stratification systems.

Design: Observational retrospective study.

Settings: A tertiary hospital in Oman.

Participants: All adult patients who underwent cardiac surgery at a major referral hospital in Oman between 2009 and 2013.

Results: $30.5 \%$ of the patients had prolonged LOS ( $\geq 11$ days) after surgery, while $17 \%$ experienced prolonged ICU LOS ( $\geq 5$ days). Factors that were identified to prolong CICU LOS were non-elective surgery, current congestive heart failure (CHF), renal failure, combined coronary artery bypass graft (CABG) and valve surgery, and other non-isolated valve or $C A B G$ surgery. Patients were divided into three groups based on their scores. The probabilities of prolonged CICU LOS were $11 \%, 26 \%$ and $28 \%$ for group 1,2 and 3 , respectively. The predictive model had an area under the curve of 0.75 . Factors associated with prolonged overall postoperative LOS included the body mass index, the type of surgery, cardiopulmonary bypass machine use, packed red blood cells use, non-elective surgery and number of complications. The latter was the most important determinant of postoperative LOS.

Conclusions: Patient management can be tailored for individual patient based on their treatments and personal attributes to optimise resource allocation. Moreover, a simple predictive score system to enable identification of patients at risk of prolonged CICU stay can be developed using data that are routinely collected by most hospitals.

\section{INTRODUCTION}

Managers and clinicians seeking to maximise resources are often interested in patient variation and how it might influence resource

\section{Strengths and limitations of this study}

- This is the first study to identify factors affecting length of stay (LOS) after a cardiac surgery in Oman, considering the unique characteristics of the local population.

- The study included a small sample size relative to other previously published models.

- Our selection of the 75th centile for defining prolonged LOS can be viewed as an arbitrary cut-off in the absence of predefined clinically acceptable value in the literature.

- The prediction model was not externally validated. Thus, the model lacks generalisability which limits its portability.

- The analysis of the association was only limited to the variables available in the hospital database and that were routinely collected.

utilisation. This is based on a basic premise that understanding the factors relating to patients, treatment and iatrogenic events on length of stay (LOS) will aid in the management of complex hospital systems. Physical limitations and government regulation can restrict expansion of hospital capacity. ${ }^{1}$ Thus, management of some of the factors that impact resource consumption can be an alternative to adding costly resources.

Prolonged LOS after cardiac surgery can have serious cost implications. Patients with high risk of prolonged LOS are an important hospital subpopulation because they tend to consume a disproportionate amount of intensive care unit (ICU) resources. ${ }^{2}$ As a result, patient variation can reduce operational performance which can manifest in the form of cancelled surgery or extended waiting lists.

In many parts of the world, critical care resources are still limited, calling for best practices in resource management. A valuable decision-making tool is to predict cardiac ICU 
(CICU) LOS in advance for every cardiac surgical patient. Therefore, an appropriate management strategy can be applied such as mitigating risk factors before surgery, instituting fast-track anaesthesia, deciding on staffing level and scheduling patients for surgery based on expected LOS. Ultimately, influential predictors of LOS can be integrated into hospital resource planning process. This can be a solution to the traditional practice of using average bed numbers as a measure of resource planning, which may not adequately reflect patient mix and fails to predict future demand. ${ }^{3}$

Several studies have identified factors associated with prolonged CICU $\operatorname{LOS}^{4-8}$ and postoperative $\operatorname{LOS}^{9}{ }^{10}$ for patients undergoing cardiac surgery. A common feature of these studies is that they are only applicable to the original study populations as they are mostly based on a single institution. No previous research was conducted to investigate factors predicting LOS after cardiac surgery in our local population. We have undertaken this study to investigate this area, considering patient and surgical unique characteristics.

\section{METHODS}

\section{Patient population and data}

We included all adult patients who underwent cardiac surgeries during the 4-year period from 2009 to 2013 at the Sultan Qaboos University Hospital (SQUH). A total of 600 consecutive patients were included. We excluded patients who died during admissions $(n=25)$ from our analysis on the basis that they will be more likely to have prolonged LOS had they survived. Data were collected prospectively during the patient's admission and entered into a database for research purpose. Postoperative outcomes included several complications, all of which are defined according to the Society of Thoracic Surgeons database definitions. ${ }^{11-13}$

The type of cardiothoracic surgery performed included isolated coronary artery bypass graft (CABG), isolated valve surgery and combined procedures. The latter category includes several complex procedures such as aortic aneurysm and aortic dissection surgery or congenital defect repair. We included them because patients who had these procedures shared the same typical resources (operating theatre, wards, etc) and from the perspective of hospital operation management, these patients compete with other patients for resources.

\section{Statistical analysis}

Continuous variables were presented as means with SD, while categorical variables were presented as frequencies. LOS at CICU and postoperative LOS were dichotomised to designate two groups (normal LOS and prolonged LOS). We defined prolonged LOS as $\geq 75$ th centile. In the absence of a prescribed LOS in the literature and to confirm the appropriateness of our selection, we consulted the surgeons for their judgement who agreed on this cut-off value. In general, there is a variability in medical research for defining the period at which a stay is considered as prolonged. ${ }^{14}$ Moreover, the use of the LOS at the 75th centile is consistent with other studies. Postoperative LOS was defined as the time between the day of surgery and discharge from the hospital, while CICU LOS was defined as the time in days between the admission and discharge from CICU. For identifying difference between groups, we used t-tests for continuous variables, Mann-Whitney for non-normally distributed variables and $\chi^{2}$ for categorical variables.

For identifying predictors of postoperative LOS, a univariate logistic regression was first performed to select variables that are significantly related to the postoperative LOS. Factors with $\mathrm{p}<0.10$ were then included in the multivariate analysis. These included sex, age, body mass index (BMI), history of diabetes, history of renal failure, history of cerebrovascular disease, history of respiratory disease, pulmonary hypertension, congestive heart failure (CHF) at current admission, preoperative arrhythmia, preoperative inotropic support, left ventricular ejection fraction ( $\geq 40 \%$ vs $<40 \%)$, preoperative haematocrit (Hct) level, non-elective surgery, type of surgery, use of cardiopulmonary bypass (CPB) machine, inotropic support after operation, use of packed red blood cells (PRBC) and number of complications. A backward stepwise logistic regression was used to identify factors that had an independent effect on the postoperative LOS. A $p$ value of 0.1 to enter a factor and 0.2 to remove it were used.

\section{The cardiac ICU prediction model}

For deriving the CICU LOS prediction scores, only preoperative factors known prior to surgery were considered. These included sex, age, BMI, body surface area (BSA), current smoking history, diabetes, renal failure, hypertension, cerebrovascular disease, peripheral vascular disease, history of respiratory disease, pulmonary hypertension, history of angina, CHF on admission, cardiogenic shock, arrhythmia, inotropic support, left main coronary artery (LMCA) disease, myocardial infarction (MI) $<24$ hours, use of thrombolysis, previous percutaneous coronary intervention (PCI), preoperative Hct level, left ventricular ejection fraction $(\geq 40 \%$ vs $<40 \%)$, non-elective surgery and type of surgery. The same process was repeated as for the first model for selecting candidate variables. The statistically significant variables $(\mathrm{p}<0.10)$ were then included in the backward stepwise logistic regression using the same entry and exit criteria as in the postoperative LOS model. A simplified score system was devised by rounding the OR of each predictor to the nearest 0.5 .

We assessed the validation and discriminatory power of the predictive model through internal validation. The models were assessed using the Hosmer-Lemeshow goodness-of-fit statistics and the receiver operating characteristic (ROC) curve. A bootstrapping on the $\beta$ coefficient and SEs was performed with 200 replications. Additionally, we compare our prediction model with 
two different cardiac risk stratification systems: the European System for Cardiac Operative Risk Evaluation (EuroSCORE) and Parsonnet score.

Assumptions were assessed using different techniques. Multicollinearity was assessed using the Pearson correlation statistics and the variance inflation factor (VIF). Highly correlated variables, for example, renal failure and dialysis, patients with diabetes and patients with insulin dependency were not combined into a single model. Moreover, influential cases are computed using Cook's distance. A case is said to be influential in logistic regression if its Cook's distance is $>1.0 .{ }^{15}$ As a rule of thumb in logistic regression, there should be at least 10 events per predictor to obtain reliable estimates of regression coefficients as suggested by Hosmer and Lemeshow. ${ }^{15}$ Inspection of candidate predictors revealed that some complications had rare events $(<10)$; consequently, they were either dropped or combined with other complications in a separate category as appropriate. All statistical analyses were performed using Stata V.12 (StataCorp LP, College Station, Texas, USA).

\section{RESULTS}

\section{Baseline and clinical characteristics}

Table 1 presents patient, surgery and LOS characteristics. The majority of patients were men (69.7\%), 13\% of the patients being older than 70 years, and there were 12 octogenarians in the dataset. The mean age of cardiac surgical patients was 58.6 years. The mean age of patients who underwent isolated CABG was 59.3, valve surgery 53, combined surgery procedures 64.2 and other types 55.3. Patients aged 40 years or younger constituted $5.6 \%$ for CABG, $5 \%$ for valve surgery, $6.7 \%$ for combined CABG plus valve surgery and $5.3 \%$ for other types of surgeries.

The 75th centiles of the CICU LOS and postoperative LOS were 5 days and 11 days, respectively. The study group used a total of 2816 cardiac ICU patients days. Patients with prolonged postoperative LOS (183 patients) had $60 \%$ of the total patients days. Only $5 \%$ of the patients who underwent cardiac surgery were discharged by the 5th postoperative day. The majority of the patients $(61 \%)$ were discharged between 6 and 10 postoperative days.

In total, $45 \%$ of the patients undergoing heart surgery had diabetes mellitus. Hypertension was prevalent in $67 \%$ of the patients. There were 229 patients who had CHF. Nearly $27 \%$ of the patients had unstable angina and $6.3 \%$ were diagnosed with atrial fibrillation. Approximately $12 \%$ of the patients had renal failure preoperatively; among these patients, only $1.3 \%$ were on dialysis. Based on BMI as used by the $\mathrm{WHO},{ }^{16}$ patients were either underweight $(<18.50)=3 \%$, normal weight $(18.50-24.99)=35 \%$, overweight $(25.00-29.99)=38 \%$ or obese $(\geq 30)=24 \%$. Male and female patients had statistically different distributions of BMI, $\mathrm{p}=0.01 \quad(\mathrm{t}=2.46)$. About $4 \%$ of the patients died after surgeries during their hospitalisation, and they were excluded from the analysis. The numbers of patients who died based on surgery type were as follows: CABG: 12, valve: 7, combined surgery: 5 and other surgeries: 1 .

The average EuroSCORE was 6.6 for all patients. The distribution of EuroSCORE was significantly different between patients with normal and prolonged LOS.

Table 2 presents the predictive factors that were significant. All variables were significant below the $\alpha$ level of 0.05 . Patients with higher BMI were more likely to experience prolonged LOS. Likewise, patients who received PRBC had 2.3 times greater likelihood of postoperative $\operatorname{LOS} \geq 11$ days $(\mathrm{OR}=2.5,95 \%$ CI 1.5 to 4.1 , $\mathrm{p}<0.01)$. The association between urgency of the surgery and the postoperative LOS remained statistically significant after adjusting for other patients' factors in the multivariate model. Non-elective surgery was associated with higher $\operatorname{LOS}(\mathrm{OR}=2.1,95 \%$ CI 1.27 to $3.2, \mathrm{p}<0.05)$. Patients who were operated with the use of CPB $(76 \%)$ had odd ratio $=2.1$ (95\% CI 1.8 to $3.9, \mathrm{p}=0.012$ ), which suggests that these patients had twice greater probability of extended stay after surgery than patients who were operated off pump. In addition to the above, patients who underwent an isolated valve or combined surgery (valve and $\mathrm{CABG}$ ) were more likely to have postoperative LOS $\geq 11$ days than patients who underwent isolated CABG.

Finally, complications were the strongest predictors of postoperative LOS. There was a stepwise risk-adjusted increase in probability of prolonged stay for 1 ( $\mathrm{OR}=2.8$, $95 \%$ CI 1.68 to $4.78, \mathrm{p}<0.01$ ), versus 2 complications $(\mathrm{OR}=5.7,95 \%$ CI 3.15 to $10.54, \mathrm{p}<0.01)$, versus 3 or more complications $(\mathrm{OR}=27,95 \%$ CI 11.56 to 62.51 , $\mathrm{p}<0.01)$. At least half of the patients experienced one or more complications. The type of complications mainly included cardiac, pulmonary, neurological and infection.

The Hosmer and Lemeshow test suggests that the model fits the data well: $c^{2}(8)=8.90 ; p=0.351$; pseudo $\mathrm{R}^{2}$ (McFadden's) $=0.27$. The model correctly classified $80.5 \%$ of the patients with prolonged LOS with 11 days or more, and finally the area under the ROC curve was 0.82 .

\section{Prediction model for CICU LOS}

The following variables emerged to be statistically significant (table 3): non-elective surgery, current chronic heart failure, renal failure, combined surgery, and other none CABG-Valve surgery. The combined surgery was the strongest predictor of prolonged LOS $(\mathrm{OR}=6,95 \%$ CI 3.3 to $10.0, \mathrm{p}<0.001)$.

To further assess the effect of including the patients who died during their hospitalisation on our results, we conducted sensitivity analysis whereby data of patients who died were included in the model. The independent risk factors identified originally remained unchanged.

The scores for the prediction model (table 4) were obtained based on the coefficients from the multivariate regression model. The scores were then assigned to each 
Table 1 Demographic and perioperative characteristics

\begin{tabular}{|c|c|c|c|c|c|c|c|}
\hline \multirow[b]{2}{*}{ Variable } & \multirow{2}{*}{$\begin{array}{l}\text { All patients } \\
(n=600)\end{array}$} & \multicolumn{3}{|c|}{ ICU LOS (days) } & \multicolumn{3}{|c|}{ Postoperative LOS (days) } \\
\hline & & $<5$ & $\geq 5$ & p Value & $<11$ & $\geq 11$ & p Value \\
\hline \multicolumn{8}{|l|}{ Continuous variables: mean \pm SD } \\
\hline $\mathrm{Age}^{\star}$ & $59 \pm 12$ & $59 \pm 12$ & $58 \pm 13$ & 0.47 & $58 \pm 12$ & $60 \pm 12$ & 0.05 \\
\hline $\mathrm{BMI}^{*}$ & $27 \pm 5.4$ & $27 \pm 5$ & $26.8 \pm 6.4$ & 0.73 & $26.7 \pm 5.3$ & $27.7 \pm 5.6$ & 0.04 \\
\hline BSA $\left(\text { in } \mathrm{m}^{2}\right)^{\star}$ & $1.71 \pm .21$ & $1.71 \pm .20$ & $1.70 \pm .21$ & 0.58 & $1.71 \pm .20$ & $1.72 \pm .21$ & 0.44 \\
\hline \multicolumn{8}{|l|}{ Length of stay } \\
\hline Total LOS & $18 \pm 17$ & $16 \pm 15$ & $27 \pm 29$ & $<0.001$ & $12.8 \pm 5$ & $31.1 \pm 29$ & $<0.001$ \\
\hline Postoperative LOS & $13 \pm 17$ & $11 \pm 12$ & $21 \pm 29$ & $<0.001$ & $7.4 \pm 2$ & $24.6 \pm 27$ & $<0.001$ \\
\hline Cumulative bypass time (in min) $\dagger$ & $118 \pm 47$ & $115 \pm 46$ & $130 \pm 50$ & 0.004 & $110.6 \pm 43$ & $132.8 \pm 51$ & $<0.001$ \\
\hline Preoperative troponin level† & $1.39 \pm 8.7$ & $81 \pm 5.3$ & $4.10 \pm 17$ & $<0.001$ & $0.94 \pm 6.7$ & $2.40 \pm 12$ & 0.02 \\
\hline EuroSCORE† & $6.6 \pm 12.3$ & $5.8 \pm 11$ & $10.2 \pm 17$ & $<0.001$ & $5.5 \pm 11$ & $9.3 \pm 15$ & $<0.001$ \\
\hline \multicolumn{8}{|l|}{ Categorical variables: $\ddagger n(\%)$} \\
\hline Female & $182(30.3)$ & $142(78)$ & $40(22)$ & 0.06 & $118(64.8)$ & $64(35.2)$ & 0.069 \\
\hline Male & $418(69.7)$ & $353(84.4)$ & $65(15.6)$ & & $302(72.2)$ & $116(27.8)$ & \\
\hline CPB use & $461(76.8)$ & $369(80)$ & $92(20)$ & 0.04 & $302(65.5)$ & 159 (34.5) & $<0.001$ \\
\hline Isolated CABG & $478(79.7)$ & $425(88.9)$ & $53(11.1)$ & $<0.001$ & $345(72.2)$ & $133(27.8)$ & 0.021 \\
\hline Combined $\mathrm{CABG}+$ valve surgery & $63(10.5)$ & $51(81)$ & $12(19)$ & 0.073 & $28(44.4)$ & $35(55.6)$ & $<0.001$ \\
\hline Isolated valve surgery & $165(27.5)$ & $109(66.1)$ & $56(33.9)$ & $<0.001$ & $90(54.5)$ & $75(45.5)$ & $<0.001$ \\
\hline Non-elective surgery & $92(15.3)$ & $68(73.9)$ & $24(26.1)$ & 0.018 & $53(57.6)$ & 39 (42.4) & 0.005 \\
\hline LVEF $<40 \%$ & $246(41)$ & $199(80.9)$ & $47(19.1)$ & 0.388 & $163(66.3)$ & $83(33.7)$ & 0.096 \\
\hline PRBC use & $374(62.3)$ & $297(79.4)$ & $77(20.6)$ & 0.010 & $231(61.8)$ & $143(38.2)$ & $<0.001$ \\
\hline Inotropic support (after surgery) & $411(74.6)$ & $329(80)$ & $82(20)$ & 0.007 & $277(67.4)$ & 134 (32.6) & 0.013 \\
\hline NYHA Class & & & & 0.004 & & & 0.003 \\
\hline 1 & $9(3.7)$ & $5(55.6)$ & $4(44.4)$ & & $7(77.8)$ & 2 (22.2) & \\
\hline 2 & $36(14.8)$ & $31(86.1)$ & $5(13.9)$ & & $28(77.8)$ & 8 (22.2) & \\
\hline 3 & $137(56.1)$ & $111(81)$ & $26(19)$ & & $96(70.1)$ & $41(29.9)$ & \\
\hline 4 & $62(25.4)$ & $38(61.3)$ & $24(38.7)$ & & $29(46.8)$ & $33(53.2)$ & \\
\hline Current smoker & $62(10.3)$ & $50(80.6)$ & $12(19.4)$ & 0.685 & $42(67.7)$ & 20 (32.3) & 0.682 \\
\hline Diabetes & $270(45)$ & $234(86.7)$ & $36(13.3)$ & 0.015 & $179(66.3)$ & $91(33.7)$ & 0.073 \\
\hline Hypercholesterolemia & $375(62.5)$ & $319(85.1)$ & $56(14.9)$ & 0.033 & $272(72.5)$ & $103(27.5)$ & 0.080 \\
\hline Renal failure & $73(12.2)$ & $48(65.8)$ & $25(34.2)$ & $<0.001$ & $39(53.4)$ & $34(46.6)$ & 0.001 \\
\hline Hypertension & $403(67.2)$ & $339(84.1)$ & $64(15.9)$ & 0.135 & $282(70)$ & $121(30)$ & 0.985 \\
\hline Cerebrovascular disease & $44(7.3)$ & $32(72.7)$ & $12(27.3)$ & 0.076 & $24(54.5)$ & $20(45.5)$ & 0.020 \\
\hline Peripheral vascular disease & $29(4.8)$ & $23(79.3)$ & $6(20.7)$ & 0.643 & $17(58.6)$ & $12(41.4)$ & 0.170 \\
\hline Pulmonary hypertension & $74(12.3)$ & $50(67.6)$ & $24(32.4)$ & $<0.001$ & $37(50)$ & $37(50)$ & $<0.001$ \\
\hline $\mathrm{Ml}<24$ hours & $21(3.5)$ & $14(66.7)$ & $7(33.3)$ & 0.052 & $14(66.7)$ & 7 (33.3) & 0.734 \\
\hline Unstable angina & $160(26.7)$ & $136(85)$ & $24(15)$ & 0.331 & $104(65)$ & $56(35)$ & 0.107 \\
\hline CHF on admission & $153(26.3)$ & $110(71.9)$ & $43(28.1)$ & $<0.001$ & $93(60.8)$ & $60(39.2)$ & 0.003 \\
\hline Preoperative arrhythmia & $72(12)$ & $52(72.2)$ & $20(27.8)$ & 0.014 & $39(54.2)$ & $33(45.8)$ & 0.002 \\
\hline Previous CV intervention & $44(7.4)$ & $34(77.3)$ & $10(22.7)$ & 0.349 & $32(72.7)$ & $12(27.3)$ & 0.671 \\
\hline LMCA disease (>50\% stenosis) & $65(11.4)$ & $58(89.2)$ & $7(10.8)$ & 0.120 & $49(75.4)$ & $16(24.6)$ & 0.304 \\
\hline
\end{tabular}

$\mathrm{BMI}$, body mass index; BSA, body surface area; CABG, coronary artery bypass graft; CHF, congestive heart failure; CPB, cardiopulmonary bypass machine; CV, cardiovascular; EuroSCORE, European System for Cardiac Operative Risk Evaluation; ICU, intensive care unit; LMCA, left main coronary artery; LOS, length of stay; LVEF, left ventricular ejection fraction; MI, myocardial infarction; NYHA, New York Heart Association; PRBC, packed red blood cells.

Test of difference between groups is based on the following:

*t-Test.

†Wilcoxon rank-sum (Mann-Whitney) test.

$\mp \chi^{2}$ test.

patient in the dataset where the highest total score was 14. Patients total scores were divided into three groups: $0-1,2-4$ and $>5$. A Kruskal-Wallis test revealed a statistically significant difference in CICU stay among the three score groups: $\chi^{2}(2)=14.19 ; \mathrm{p}<0.001$. The average CICU LOS was 4, 5 and 6.5 days for the first, second and third score groups, respectively. The probabilities of prolonged CICU LOS were 11\%, 26\% and 28\% for group 1, 2 and 3 , respectively.
The predictive score model was tested by means of Hosmer-Lemeshow and ROC curve. The model demonstrated a good discrimination according to the Hosmer-Lemeshow test: $c^{2}(8)=3.18 ; p=0.922$. The area under the ROC curve was found to be 0.75 ( $\mathrm{SE}=0.027$ ).

Since the first 200 patients were scored using Parsonnet score, we restricted the comparison of the three scoring systems to these 200 patients. Our simple model compared favourably to the logistic EuroSCORE 
Table 2 Predictors of prolonged postoperative LOS

\begin{tabular}{|c|c|c|}
\hline Variables & OR & SE \\
\hline \multicolumn{3}{|l|}{ Patient factors } \\
\hline BMI & $1.076^{\star \star \star}$ & $(0.022)$ \\
\hline \multicolumn{3}{|l|}{ Surgical factors } \\
\hline \multicolumn{3}{|l|}{ Type of surgery* } \\
\hline Valve & $3.034^{\star * *}$ & $(0.904)$ \\
\hline Combined valve and CABG & $2.062^{\star *}$ & $(0.733)$ \\
\hline CPB use & $2.152^{\star \star}$ & $(0.660)$ \\
\hline PRBC & $2.521^{\star * \star}$ & $(0.631)$ \\
\hline Non-elective surgery & $2.123^{\star \star}$ & $(0.630)$ \\
\hline \multicolumn{3}{|l|}{ Number of complications $†$} \\
\hline One complication & $2.843^{\star \star \star}$ & $(0.756)$ \\
\hline Two complications & $5.714^{\star \star \star}$ & $(1.786)$ \\
\hline Three or more complications & $26.89^{\star \star \star}$ & (11.57) \\
\hline Constant & $0.003^{* * *}$ & (0.002) \\
\hline
\end{tabular}

${ }^{* * *} \mathrm{p}<0.01 ;{ }^{* *} \mathrm{p}<0.05 ;{ }^{*} \mathrm{p}<0.1$

*Omitted reference category is 'CABG'.

†Omitted reference category is 'no complications'.

$\mathrm{BMI}$, body mass index; CABG, coronary artery bypass graft; CPB, cardiopulmonary bypass; LOS, length of stay; PRBC, packed red blood cells.

and the additive Parsonnet. The areas under the curve were 0.75 for our model, 0.70 for the EuroSCORE and 0.65 for the Parsonnet score.

\section{DISCUSSION}

We developed two models to identify factors predictive of prolonged LOS. The first model identified predictive factors for the overall postoperative LOS. We also derived a second model to predict prolonged LOS in the CICU, a major bottleneck in many hospitals. The good performance of these two models was evident by the good discriminative power $(\mathrm{AUC}=0.82$ and 0.75 , for the overall postoperative LOS and for the CICU LOS models, respectively).

An early recognition of patients at risk of prolonged LOS was the main objective of several studies. ${ }^{17}$ Some applications of stratifying patients based on their expected LOS include identifying patients that can be selected for fast-track protocols to minimise ICU stay or bypass it altogether, ${ }^{18}$ providing anaesthesiologists with enough time to correct risk factors ${ }^{19}$ and facilitating the

Table 3 Preoperative variables predicting CICU LOS

\begin{tabular}{lll}
\hline Variables & OR & SE \\
\hline Non-elective surgery & $1.779^{\star}$ & $(0.545)$ \\
Current CHF & $1.894^{\star *}$ & $(0.482)$ \\
Renal failure & $4.015^{\star * *}$ & $(1.268)$ \\
Combined valve and CABG surgery & $5.835^{\star * *}$ & $(1.610)$ \\
Other surgery type & $5.067^{\star * *}$ & $(2.760)$ \\
Constant & $0.079^{* \star *}$ & $(0.016)$ \\
\hline${ }^{* \star *} p<0.01 ;{ }^{* *} p<0.05 ;{ }^{*} p<0.1$. & \\
CABG, coronary artery bypass graft; CHF, congestive heart \\
failure; CICU, cardiac intensive care unit; LOS, length of stay.
\end{tabular}

Table 4 Predictive score for length of CICU stay

\begin{tabular}{ll}
\hline Variables & Score \\
\hline Surgery urgency level & \\
Elective & 0 \\
Non-elective surgery & 2 \\
Current CHF & 2 \\
Renal failure & 4 \\
Type of surgery & \\
Isolated CABG or isolated valve & 0 \\
Combined valve and CABG surgery & 6 \\
Other surgery types & 5 \\
\hline
\end{tabular}

CABG, coronary artery bypass graft; $\mathrm{CHF}$, congestive heart failure; $\mathrm{CICU}$, cardiac intensive care unit.

selection of resource planning strategy based on patient risk of prolonged LOS. With the current economic climate in Oman, hospitals are left with no option but to efficiently manage their resources. We believe much of this efficiency can be accomplished through proper management of care process for patients at risk of prolonged stay.

Even in a relatively homogenous group such as cardiac surgical patients, we found wide variation in resource use. An implication of this to hospital planners is that resource allocation should be planned based on individual patient characteristics. Factors related to patients and treatments that are known to prolong postoperative LOS can be valuable information for planning hospital staff and beds.

Our findings are consistent with previous studies that have explored postoperative LOS after cardiac surgery. For example, combined cardiac surgery was predictive of prolonged LOS. ${ }^{20}$ The use of CPB was also found to be associated with longer hospitalisation. ${ }^{14}{ }^{21-24}$ The conclusion that can be drawn from these studies is that on-pump patients were more likely to stay in hospital longer and develop complications compared with patients operated without the use of CPB machine. In general, complications are known to prolong LOS following cardiac surgery. For example, new onset of atrial fibrillation, ${ }^{25-27}$ renal dysfunction ${ }^{28}$ and deep sternal wound infection ${ }^{29}$ were associated with increased ICU and postoperative LOS. We found that the hospital stay increased monotonically with the number of complications.

The incremental cost associated with complications such as septicaemia or stroke can be substantial. ${ }^{30}$ In the hospital under study, a higher number of patients experienced postoperative complications (at least half of the patients). In reality, many of the complications encountered by the patients such as infections are preventable. ${ }^{31}$ Quality and safety initiatives aimed at reducing the number of complications can substantially lower hospital cost by indirectly reducing LOS.

Despite the high prevalence of high lifestyle diseases among the Omani population ${ }^{32}$ and among patients in our dataset, diabetes and hypertension were not 
predictors of prolonged LOS. In contrast to our findings, diabetes in particular was found to be a predictor of prolonged LOS among patients with CABG in previous studies. ${ }^{33} 34$

While the available capacity in a cardiothoracic ward can affect cardiac patient discharge rate from the CICU, ${ }^{35}$ this was not the case in the hospital under study as sufficient number of beds were allocated in the ward. If the downstream capacity was limited, we would expect a higher proportion of patients to have prolonged LOS for this system-related reason which can impact on our results.

It is worth mentioning that patients in our sample had considerably higher overall postoperative LOS in comparison with what has been found in other studies. ${ }^{10} 36$ Consequently, the benefits of risk-stratifying patients based on their expected LOS for optimising resources can be outweighed by the inefficiency in the use of hospital beds introduced by factors unrelated to patient condition such as hospital discharge policy and physician judgement. This should be considered in future studies investigating allocation of resources based on influential factors related to patients.

\section{The use of the CICU prediction model}

We developed an objective scoring system, yet simple, that was able to reasonably predict prolonged CICU stay based on a few preoperative variables. Previous research has suggested the use of existing risk stratification systems such as the EuroSCORE and the Parsonnet for predicting ICU duration. ${ }^{37}$ Originally, these scoring systems were designed as a prognostic tool to predict mortality. Even though the EuroSCORE has all the variables that we found to be predictive of CICU LOS, this scoring system might not be in use in many hospitals (including the other hospital authorised to perform cardiac surgery in Oman). Moreover, the amount of data (and their availability) needed for calculating the EuroSCORE can be a precluding factor of its use. Thus, a prediction model based on smaller number of variables, like the one we proposed, can be of value to clinicians and bed managers who do not have sufficient data to build full-risk models.

Non-elective surgery and the type of surgery were independently significant in both models. Contrary to some previous studies that have found age ${ }^{39-41}$ and $\operatorname{sex}^{541}$ to be risk factors for prolonged CICU stay, these two basic demographic variables were not statistically significant in our models. Renal failure and renal dysfunction were both previously linked to increased CICU stay after cardiac surgery. ${ }^{34}{ }^{41-43} \mathrm{CHF}$ at admission was only significant in the CICU model. This is consistent with other studies. ${ }^{18} 40$

When compared with previously published CICU prediction models, all of the predictors in our study have been reported before. However, these models differ considerably in their type of predictors. For example, Messaoudi $e t a l^{17}$ reported in a systematic review that the number of predictors among the reviewed studies ranged from 1 to 16 (with an average of 6 predictors). With such variation surrounding the selection of predictors among several studies, it would be inaccurate and misguiding to assume a model that was developed in one population would be valid for another. Therefore, the type of predictors (and the model) in our study should be relevant to the Omani hospitals and might be also applicable to other Gulf States. Moreover, unlike other studies which introduced models with many predictors, our finding suggests that predicting CICU LOS can be possible with fairly small number of predictors.

\section{Implications for hospital resource planning}

Resource planning can be more effective if factors contributing to high resource use are appropriately managed. Clinicians can initiate preventive measures through aggressive treatment to reduce risk factors prior to surgery. A small reduction in LOS will result in a large cost saving. Risk stratification can be used to evaluate the appropriate patient management strategies (eg, aggressive treatment of comorbidities), to communicate the likelihood of CICU LOS to the patient, to aid in scheduling surgery or to be used when comparing CICU patients between hospitals.

\section{STUDY LIMITATIONS}

Our study has some limitations that merit a discussion. First, the prediction model was not externally validated. Thus, the model lacks generalisability which limits its portability to other settings. Nevertheless, it is worth mentioning that a universal LOS prediction model may also be difficult to develop because LOS distributions depend on institutional policies which greatly influence discharge practices. ${ }^{40}$ Second, the study included a small sample size relative to other previously published models. Despite this limitation, our dataset had some rare events such as stroke, renal failure and pulmonary hypertension that may not be expected to be captured in small datasets. Third, we acknowledge that there are some other non-clinical factors that might have influenced LOS but not included in our models due to unavailability of data. Such factors include physicians' judgements, hospital policy and demand and capacity considerations. Finally, our selection of the 75th centile for defining prolonged LOS can be viewed as an arbitrary cut-off in the absence of predefined clinically acceptable value in the literature.

Contributors AA, PA and HA designed the study. AA performed the literature review, wrote the first draft of the manuscript and conducted the statistical analysis. HA directed the collection of the data. MM and BB performed the data collection and interpretation of the results. PA appraised the study quality and assisted in statistical analysis. All authors contributed to drafts of the manuscript and approved the final manuscript.

Funding This research was part of AA's PhD research which was generously funded by the government of Oman.

Competing interests None declared. 
Ethics approval Ethical approval to conduct the study was granted by the hospital and the research ethical committee of the Sultan Qaboos University.

Provenance and peer review Not commissioned; externally peer reviewed.

Data sharing statement No additional data are available.

Open Access This is an Open Access article distributed in accordance with the Creative Commons Attribution Non Commercial (CC BY-NC 4.0) license, which permits others to distribute, remix, adapt, build upon this work noncommercially, and license their derivative works on different terms, provided the original work is properly cited and the use is non-commercial. See: http:// creativecommons.org/licenses/by-nc/4.0/

\section{REFERENCES}

1. Bazzoli GJ, Brewster LR, May JH, et al. The transition from excess capacity to strained capacity in U.S. hospitals. Milbank $Q$ 2006:84:273-304

2. Schoffelen A, Hofhuis J, Rommes J, et al. Consumption of ICU resources by long-stay patients does not change over time: 10-year observation in a teaching hospital in The Netherlands. Critical Care 2008;12(suppl 2):P525.

3. Rechel B, Wright S, Barlow J, et al. Hospital capacity planning: from measuring stocks to modelling flows. Bull World Health Organ 2010;88:632-6.

4. Abrahamyan L, Demirchyan A, Thompson ME, et al. Determinants of morbidity and intensive care unit stay after coronary surgery. Asian Cardiovasc Thorac Ann 2006;14:114-18.

5. Cacciatore F, Belluomo Anello C, Ferrara N, et al. Determinants of prolonged intensive care unit stay after cardiac surgery in the elderly. Aging Clin Exp Res 2012;24:627-34.

6. Azarfarin R, Ashouri N, Totonchi Z, et al. Factors influencing prolonged ICU stay after open heart surgery. Res Cardiovasc Med 2014;3:e20159.

7. Nakasuji M, Matsushita M, Asada A. Risk factors for prolonged ICU stay in patients following coronary artery bypass grafting with a long duration of cardiopulmonary bypass. J Anesth 2005;19:118-23.

8. Hörer J, Eicken A, Müller S, et al. Risk factors for prolonged intensive care treatment following atrial septal defect closure in adults. Int J Cardiol 2008;125:57-61.

9. Sokolovic E, Schmidlin D, Schmid ER, et al. Determinants of costs and resource utilization associated with open heart surgery. Eur Heart J 2002;23:574-8.

10. Atoui $\mathrm{R}, \mathrm{Ma} \mathrm{F}$, Langlois $\mathrm{Y}$, et al. Risk factors for prolonged stay in the intensive care unit and on the ward after cardiac surgery. $J$ Card Surg 2008;23:99-106.

11. O'Brien SM, Shahian DM, Filardo G, et al. The Society of Thoracic Surgeons 2008 cardiac surgery risk models: part 2-isolated valve surgery. Ann Thorac Surg 2009;88:S23-42.

12. Shahian DM, O'Brien SM, Filardo G, et al. The Society of Thoracic Surgeons 2008 cardiac surgery risk models: part 1-coronary artery bypass grafting surgery. Ann Thorac Surg 2009;88:S2-S22.

13. Shahian DM, O'Brien SM, Filardo G, et al. The Society of Thoracic Surgeons 2008 cardiac surgery risk models: part 3-valve plus coronary artery bypass grafting surgery. Ann Thorac Surg 2009;88: S43-62.

14. Bucerius J, Gummert JF, Walther T, et al. Predictors of prolonged ICU stay after on-pump versus off-pump coronary artery bypass grafting. Intensive Care Med 2004;30:88-95.

15. Hosmer DW Jr, Lemeshow S, Sturdivant RX. Applied logistic regression. John Wiley \& Sons, 2013.

16. WHO. BMI classification Secondary BMI classification 2015. http:// apps.who.int/bmi/index.jsp?introPage=intro 3.html

17. Messaoudi N, De Cocker J, Stockman B, et al. Prediction of prolonged length of stay in the intensive care unit after cardiac surgery: the need for a multi-institutional risk scoring system. J Card Surg 2009;24:127-33.

18. Wagener G, Minhaz M, Wang S, et al. The Surgical Procedure Assessment (SPA) score predicts intensive care unit length of stay after cardiac surgery. J Thorac Cardiovasc Surg 2011;142:443-50.

19. Tribuddharat S, Sathitkarnmanee T, Ngamsangsirisup K, et al. Development of an open-heart intraoperative risk scoring model for predicting a prolonged intensive care unit stay. BioMed Res Int 2014;2014:158051.
20. Lazar HL, Fitzgerald C, Gross S, et al. Determinants of length of stay after coronary artery bypass graft surgery. Circulation 1995;92:20-4

21. Welsby IJ, Bennett-Guerrero E, Atwell D, et al. The association of complication type with mortality and prolonged stay after cardiac surgery with cardiopulmonary bypass. Anesth Analg 2002;94:1072-8.

22. Utriyaprasit K. Relationship between selected factors and length of hospital stay in coronary artery bypass graft patients. Siriraj Med J 2011;63(2):52-7.

23. Scott BH, Seifert FC, Grimson R, et al. Resource utilization in on-and off-pump coronary artery surgery: factors influencing postoperative length of stay-an experience of 1,746 consecutive patients undergoing fast-track cardiac anesthesia. J Cardiothorac Vasc Anesth 2005;19:26-31.

24. Boyd WD, Desai ND, Del Rizzo DF, et al. Off-pump surgery decreases postoperative complications and resource utilization in the elderly. Ann Thorac Surg 1999;68:1490-3.

25. LaPar DJ, Speir AM, Crosby IK, et al. Postoperative atrial fibrillation significantly increases mortality, hospital readmission, and hospital costs. Ann Thorac Surg 2014;98:527-33.; discussion 33

26. Hravnak M, Hoffman LA, Saul MI, et al. Resource utilization related to atrial fibrillation after coronary artery bypass grafting. Am J Crit Care 2002;11:228-38.

27. Shirzad M, Karimi A, Tazik M, et al. Determinants of postoperative atrial fibrillation and associated resource utilization in cardiac surgery. Rev Esp Cardiol 2010;63:1054-60.

28. Mangano CM, Diamondstone LS, Ramsay JG, et al. Renal dysfunction after myocardial revascularization: risk factors, adverse outcomes, and hospital resource utilization. The Multicenter Study of Perioperative Ischemia Research Group. Ann Intern Med 1998;128:194-203.

29. Hollenbeak CS, Murphy DM, Koenig S, et al. The clinical and economic impact of deep chest surgical site infections following coronary artery bypass graft surgery. Chest 2000;118:397-402.

30. Iribarne A, Burgener JD, Hong K, et al. Quantifying the incremental cost of complications associated with mitral valve surgery in the United States. J Thorac Cardiovasc Surg 2012;143:864-72.

31. Wendler O, Baghai M. Infections post-cardiac surgery: new information during challenging times. J Am Coll Cardiol 2014;64:382-4.

32. Al Riyami A, Elaty MAA, Morsi M, et al. Oman World Health Survey: part 1-Methodology, sociodemographic profile and epidemiology of non-communicable diseases in Oman. Oman Med $J$ 2012;27:425-43.

33. Oliveira EK, Turquetto AL, Tauil PL, et al. Risk factors for prolonged hospital stay after isolated coronary artery bypass grafting. Rev Bras Cir Cardiovasc 2013;28:353-63.

34. Ghotkar SV, Grayson AD, Fabri BM, et al. Preoperative calculation of risk for prolonged intensive care unit stay following coronary artery bypass grafting. J Cardiothorac Surg 2006;1:14.

35. Bowers J. Balancing operating theatre and bed capacity in a cardiothoracic centre. Health Care Manag Sci 2013;16:236-44.

36. Cowper PA, DeLong ER, Hannan EL, et al. Trends in postoperative length of stay after bypass surgery. Am Heart J 2006;152:1194-200.

37. Lawrence DR, Valencia O, Smith EE, et al. Parsonnet score is a good predictor of the duration of intensive care unit stay following cardiac surgery. Heart 2000;83:429-32.

38. Nilsson J, Algotsson L, Höglund $\mathrm{P}$, et al. EuroSCORE predicts intensive care unit stay and costs of open heart surgery. Ann Thorac Surg 2004;78:1528-34.

39. Rosenfeld R, Smith JM, Woods SE, et al. Predictors and outcomes of extended intensive care unit length of stay in patients undergoing coronary artery bypass graft surgery. J Card Surg 2006;21:146-50.

40. Widyastuti $Y$, Stenseth $R$, Wahba $A$, et al. Length of intensive care unit stay following cardiac surgery: is it impossible to find a universal prediction model? Interact Cardiovasc Thorac Surg 2012;15:825-32

41. De Cocker J, Messaoudi N, Stockman BA, et al. Preoperative prediction of intensive care unit stay following cardiac surgery. Eur $J$ Cardiothorac Surg 2011;39:60-7.

42. Scott BH, Seifert FC, Grimson R, et al. Octogenarians undergoing coronary artery bypass graft surgery: resource utilization, postoperative mortality, and morbidity. J Cardiothorac Vasc Anesth 2005;19:583-8.

43. Giakoumidakis K, Baltopoulos GI, Charitos C, et al. Risk factors for prolonged stay in cardiac surgery intensive care units. Nurs Crit Care 2011;16:243-51. 